\title{
A Novel Self-adaptive Fuzzy-PID Controller for Temperature Control in Variable Refrigerant Volume (VRV) Air Conditioning Systems
}

\author{
Jinqiang $\mathrm{Xu}^{1,2,3}$ Ziping Feng ${ }^{1}$ \\ ${ }^{1}$ Chinese Academy of Sciences Guangzhou Institute of Energy Conversion, Guangzhou 510640, P.R. China \\ ${ }^{2}$ Graduate School of Chinese Academy of Sciences, Beijing 100049, P.R. China \\ ${ }^{3}$ Guangdong Ocean University Information College, Zhanjiang 524088, P.R. China
}

\begin{abstract}
Considering the multi-variable, nonlinearity, time lag and time variation characters of Variable Refrigerant Volume (VRV) air conditioning systems, a robust and self-adaptive two-stage Fuzzy Proportional Integral Derivative (FPID) controller for temperature control in a triple evaporator VRV system is proposed and simulated. The controller combines the Fuzzy-PD controller and Fuzzy-I controller with a fuzzy switch. The fuzzy-PD controller is employed to decrease the overshoot and rise time during the transient response period and the fuzzy-I controller is used to get disturbance rejection and zero steady state error. Obtained results show that the proposed controller not only is robust, but also it gives excellent dynamic and steady-state characteristics compared with traditional controllers.
\end{abstract}

Keywords: VRV systems, Two-Stage Fuzzy-PID, Temperature control, Robust and Self-adaptive, Simulation.

\section{Introduction}

It is well known that the overall dynamics of Variable Refrigerant Volume (VRV) air conditioning systems which consist of many mechanical and electrical components are highly nonlinear. The nonlinearities are difficult to realize with conventional model based linear controller such as PID. The conventional Fuzzy Logic Controller (FLC) is easy to perform in industry due to its simple control structure, ease of design and inexpensive cost [1]. However, FLC with fixed scaling factors and fuzzy rules may not provide perfect control performance if the controlled plant is highly nonlinear and uncertain [1]. And conventional FLC may result in steady state errors if the system does not have an inherent integrating property [2].

In order to improve conventional FLC and PID controller, some controllers have been proposed in the literature such as Fuzzy-PID (FPID) controller [3]-[5]. It should be pointed out that classical FPID controller requires a three-dimensional rule base which makes the design process more difficult. To overcome this drawback and focus on separation of the PD part from the integral part, this paper presents a two-stage FPID controller with fuzzy switch and uses it to control the room temperature in a triple evaporator VRV system. This controller uses two-dimensional inference engine (rule base) to perform reasonably the task of a threedimensional controller [6]-[8]. So it requires fewer resources to operate and its role in the system response is more apparent. In order to evaluate the effectiveness of the proposed controller some simulations are given and the obtained results show that the proposed controller not only is robust, but also it gives excellent dynamic and steady-state characteristics compared with traditional controllers.

\section{Temperature control strategies}

The triple evaporator VRV system considered is charged with R22. It consists of three indoor units and one outdoor unit. The outdoor unit is mainly composed of a variable-speed compressor, a condenser and a variable speed fan. Each indoor unit is mainly composed of an evaporator, a three-speed fan and an electronic expansion valve. A conceptual scheme of the triple evaporator VRV air conditioning system is shown in Fig. 1.

The objectives of the controller for an air conditioning system are basically to maintain the desired indoor air temperatures in air conditioned rooms at their set points and to minimize its energy consumption under various operating conditions. In order to realize the objectives, the mass flow rate of refrigerant entering each evaporator must vary in accordance with the required cooling capacity. However, in a VRV system, multiple evaporators with different capacities and operating conditions share a common suction pressure of refrigerant before the 
compressor and a common condensing pressure before the electronic expansion valves. The operating parameters, such as evaporating pressure and refrigerant flow rate in one of evaporators can certainly interfere with those of the others. Simultaneously, the compressor capacity should be regulated to match the total thermal load from the three conditioned rooms. This not only can help reduce the fluctuation of air temperature inside the conditioned space but also can improve the energy efficiency ratio, particularly during part load operation.

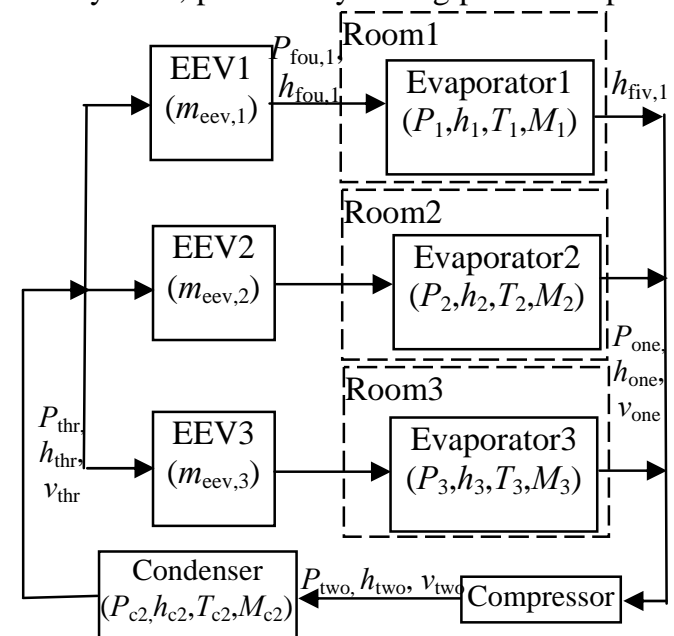

Fig.1: Conceptual scheme of triple evaporator VRV system

It has been found in previously reported work that if the suction pressure of a VRV system can be somehow maintained at a certain value, the interference among the evaporators can be restrained [9]. The variation of the suction pressure before the compressor can also reflect the variation of the total thermal load imposed on the VRV system. Under a constant speed of compressor, when the thermal load goes beyond the cooling capacity of the VRV, the suction pressure should increase. Adversely, the suction pressure will decrease. Therefore, regulating the speed of compressor to maintain the suction pressure of a VRV at a certain value, the following control strategies for air conditioned rooms are proposed [10].

The refrigerant mass flow rate entering an individual evaporator is separately controlled by an individual electronic expansion valve (EEV). An EEV has advantages such as a flexible sampling method, sensitive response, programmed control action, etc. Therefore, it is particularly suitable for use in a VRV. The use of an EEV can remove the constraint that the control of the expansion valve opening always depends on the refrigerant degree of superheat. Therefore, unlike the conventional control of the expansion device used in a variable-speed air conditioning system, the room temperature is taken as the control signal for an EEV in a VRV, as follows:

(a) When the room air temperature is higher than its upper limit, maximum of the throttling range, the EEV opens fully.

(b) When the room air temperature is within the limits of its throttling range, the temperature error (e)between the room air temperature and its setting, the change rate of the error $\left(d_{e}\right)$ and the integral value of the error $\left(R_{e}\right)$ determine the action of the EEV via a two-stage FPID controller, which will be discussed later.

(c) When the room air temperature is below its lower limit, minimum of the throttling range, the EEV is modulated to operate at the minimum opening.

\section{Modeling of the VRV system}

In this paper, EEV is regarded as actuator and its opening as the control variable. Simultaneously, the conditioned room is regarded as plant and its temperature as the controlled variable. Here introducing the EEV and conditioned room model, the corresponding dynamic model such as compressor, evaporator and condenser refer to literature [11].

\subsection{Electronic expansion valve}

In EEV1, EEV2 and EEV3, the mass flow rates through the EEVs are represented by the orifice equation. Taking EEV1 as an example, its sub-model can be expressed as follows:

$$
\begin{gathered}
m_{e e v, 1}=A_{e e v, 1} \cdot \zeta \cdot \sqrt{\frac{\left(P_{t h r}-P_{f o u, 1}\right)}{v_{t h r}}} \\
h_{f o u, 1}=h_{t h r}
\end{gathered}
$$

Where $m$ is refrigerant mass flow rate, $\mathrm{kg} / \mathrm{s}, A$ is the heat transfer area, $\mathrm{m}^{2}, \zeta$ is valve flow coefficient, $P$ is pressure, $\mathrm{Pa}, v$ is specific volume, $\mathrm{m}^{3} / \mathrm{kg}, h$ is enthalpy, $\mathrm{kJ} / \mathrm{kg}$.

\subsection{Conditioned room}

For the air conditioned rooms represented by Room1, Room2 and Room3, it is assumed that no outdoor air is supplied to each room, and the internal heat gain from both people and equipment is represented by a constant internal thermal load, $Q_{\text {indoor }}$. Therefore, the total thermal load of each room included both internal thermal load and the heat transfer between the indoor and outdoor air through the wall. Taking Room 1 as an example, the energy balance of the room yielded: 


$$
\begin{aligned}
& \left(C_{o, 1}+\rho_{\text {air }} C_{p, a i r} S_{1}\right) \frac{d T_{1}}{d t} \\
& =U_{w, 1} A_{w, 1}\left(T_{a m b}-T_{1}\right)+Q_{\text {indoor }}-Q_{\text {Evaporator } 1}
\end{aligned}
$$

Where $C_{\mathrm{o}}$ is room heat capacity, $\mathrm{kJ} / \mathrm{K}, \rho$ is density, $\mathrm{kg} / \mathrm{m}^{3}, C_{\mathrm{p}}$ is specific heat, $\mathrm{kJ} / \mathrm{kg} . \mathrm{K}, S_{1}$ is space volume of room, $\mathrm{m}^{3}, T$ is room temperature, $\mathrm{K}, U_{\mathrm{w}, 1}$ is wall heat transfer coefficient, $\mathrm{kW} / \mathrm{m}^{2}, A_{\mathrm{w}, 1}$ is wall heat transfer area, $\mathrm{m}^{2}, T_{\mathrm{amb}}$ is temperature of ambient environment.

\section{Two-stage FPID controller design}

A two-stage FPID controller combines the fuzzy PD controller and the integral controller with a fuzzy switch. The fuzzy PD stage is employed to penalize fast change and large overshoot in the control input due to corresponding practical constraints. The integral stage is used to get disturbance rejection and zero steady state error. The structure of the two-stage FPID controller is shown in Fig.2.In the two-stage structure, input values are converted to truth value vectors and applied to their respective rule base. The output fuzzy vectors are not defuzzified to crisp values as with a single stage fuzzy logic controller but are passed on to the next stage as a truth-value vector input[7]. The darkened lines in Fig.2 indicate fuzzy vectors.

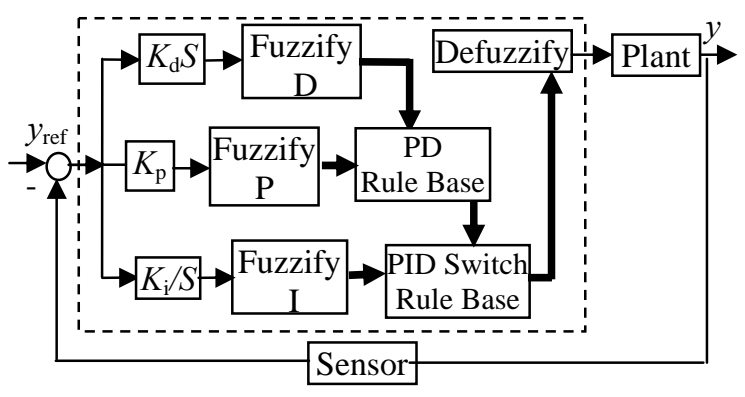

Fig.2: Structure of the proposed FPID controller

In this effort, all membership functions are defined as triangular partitions with seven segments from -3 to 3 . Zero (ZE) is the center membership function, which is centered at zero. The partitions are also symmetric about the ZE membership function as shown in Fig.3. The remaining parts of the partition are negative big (NB), negative medium (NM), negative small (NS), positive small (PS), positive medium (PM) and positive big (PB).

There are two rule bases used in two-stage FPID controller. The first is called the PD rule base as it operates on truth vectors inputs from the temperature error $(e)$ and change rate of error $(d e)$, the output is the truth vectors of the EEV opening. A typical PD rule base for the fuzzy logic controller is given in Table 1.

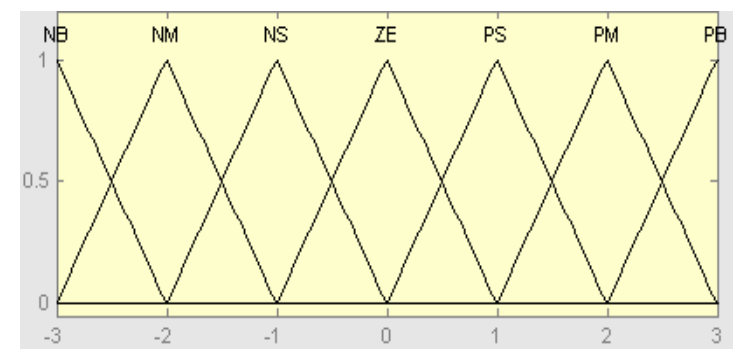

Fig. 3: Membership functions

Table 2 shows a PID switch rule base. This rule base is designed to pass through the PD input if the PD input is not in the zero fuzzy set. If the PD input is in the zero fuzzy set, then the PID switch rule base passes the integral value of temperature error $(R e)$. This rule base operates as the behavior switch, giving control to PD feedback when the system is in motion and reverting to integral feedback to remove the steady state error when the system is no longer moving. The

\begin{tabular}{|c|c|c|c|c|c|c|c|c|}
\hline & \multicolumn{7}{|c|}{$d e$} \\
\hline & & $N B$ & $N M$ & $N S$ & $Z E$ & $P S$ & $P M$ & $P B$ \\
\hline \multirow{7}{*}{$e$} & $N B$ & $N B$ & $N B$ & $N B$ & $N B$ & $N M$ & $N S$ & $Z E$ \\
\hline & $N M$ & $N B$ & $N B$ & $N B$ & $N M$ & $N S$ & $Z E$ & $P S$ \\
\hline & $N S$ & $N B$ & $N B$ & $N M$ & $N S$ & $Z E$ & $P S$ & $P M$ \\
\hline & $Z E$ & $N B$ & $N M$ & $N S$ & $Z E$ & $P S$ & $P M$ & $P B$ \\
\hline & $P S$ & $N M$ & $N S$ & $Z E$ & $P S$ & $P M$ & $P B$ & $P B$ \\
\hline & $P M$ & $N S$ & $Z E$ & $P S$ & $P M$ & $P B$ & $P B$ & $P B$ \\
\hline & $P B$ & $Z E$ & $P S$ & $P M$ & $P B$ & $P B$ & $P B$ & $P B$ \\
\hline
\end{tabular}
output truth vectors are the same as PD rule base.

Table1: PD rule base

The operation used to determine the consequence value at the intersection of two input fuzzy values is given as:

$$
c_{i, j}=a_{i} * b_{j}
$$

Where $a_{i}$ is the membership value of the $i$ th fuzzy set for a given $e$ input, $b_{j}$ is likewise for a given $d e$ input, and $i, j=1,2 \ldots .7$.

The operation used to determine the membership value of the $k$ th consequence set is:

$$
C_{k}=\sum c\left(u_{k}\right) / u_{k}
$$

\begin{tabular}{|c|c|c|c|c|c|c|c|c|}
\hline & & \multicolumn{7}{|c|}{ Fuzzy-PD values } \\
\hline & & $N B$ & $N M$ & $N S$ & $Z E$ & $P S$ & $P M$ & $P B$ \\
\hline \multirow{7}{*}{$R e$} & $N B$ & $N B$ & $N M$ & $N S$ & $N B$ & $P S$ & $P M$ & $P B$ \\
\hline & $N M$ & $N B$ & $N M$ & $N S$ & $N M$ & $P S$ & $P M$ & $P B$ \\
\hline & $N S$ & $N B$ & $N M$ & $N S$ & $N S$ & $P S$ & $P M$ & $P B$ \\
\hline & $Z E$ & $N B$ & $N M$ & $N S$ & $Z E$ & $P S$ & $P M$ & $P B$ \\
\hline & $P S$ & $N B$ & $N M$ & $N S$ & $P S$ & $P S$ & $P M$ & $P B$ \\
\hline & $P M$ & $N B$ & $N M$ & $N S$ & $P M$ & $P S$ & $P M$ & $P B$ \\
\hline & $P B$ & $N B$ & $N M$ & $N S$ & $P B$ & $P S$ & $P M$ & $P B$ \\
\hline
\end{tabular}

Where $u_{k}$ is the output linguistic values, and $k=1,2 \ldots .7$.

Table2: PID switch rule base 
The defuzzification uses the weighted average method where $O U T_{k}$ is the crisp value of the $k$ th output

$$
O U T_{k}=\frac{\sum\left(c\left(u_{k}\right)^{*} u_{k}\right)}{\sum c\left(u_{k}\right)}
$$

\section{Simulation results and discuss}

Taking the air-conditioned Room1 introduced in section 2 as example to study the steady state and transient characteristics of the proposed control strategies incorporating the two-stage FPID controller. At the same time, we compare the results with the conventional controller such as FLC and PID. Here assuming the outdoor ambient air temperature is $36^{\circ} \mathrm{C}$, and the room air temperature setting is $26^{\circ} \mathrm{C}$.

\subsection{Set point tracking performance}

The simulation results of the VRV system under twostage FPID, FLC and PID controllers are shown in Fig.4. It can be seen that the room air temperature under three controllers approached and stabilized at the setting point. But the overshoot of the temperature transient response under PID controller is relatively large and the oscillation is more intense, the oscillation under FLC controller is not intense relatively but the response is slow and the settling time is long. On the contrary, under two-stage FPID controller the transient response has better performance at settling time and maximum overshoot, i.e. system under such controller attains balance between the less initial energy and faster response.

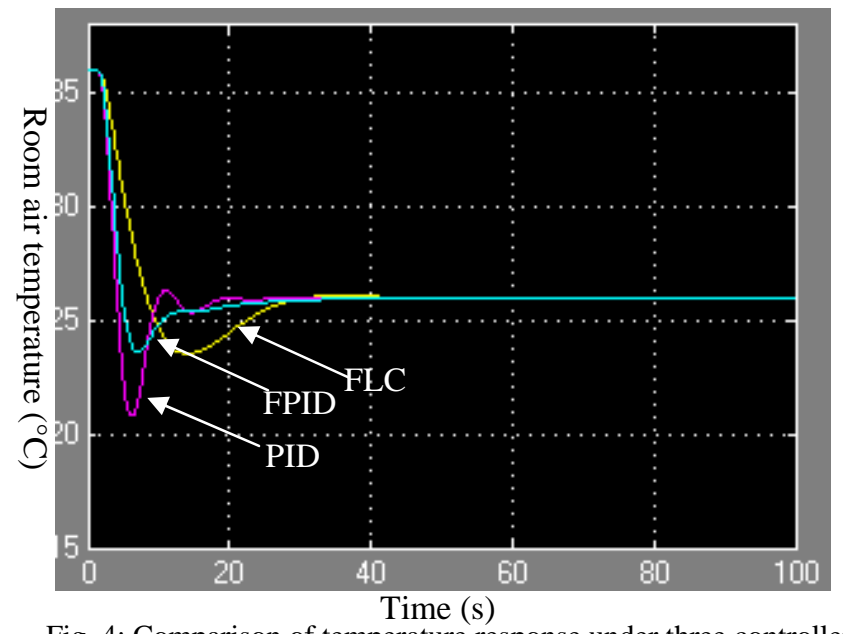

Fig. 4: Comparison of temperature response under three controllers

\subsection{Robustness analysis}

Input and outer disturbance, system structure and parameter variation are the main measures to see about system robustness performance. In this paper, outer periodic sine disturbance signal is used to analyze the robustness of the three controllers. The sine disturbance signal is simplified as $0.05 * \sin 10 t$ and the simulation results are shown in Fig.5. It can be seen that under the three controllers system can attain stability finally although the transient responses are influenced by the sine disturbance signal more or less. The influence by disturbance under PID controller is much more than that of FLC and two-stage FPID controllers. The variation of response under two-stage FPID controller may be ignored at certain error range. Further research shows that the robustness of the twostage FPID control system is also excellent when the periodic pulse disturbance signal occurs at the input port.

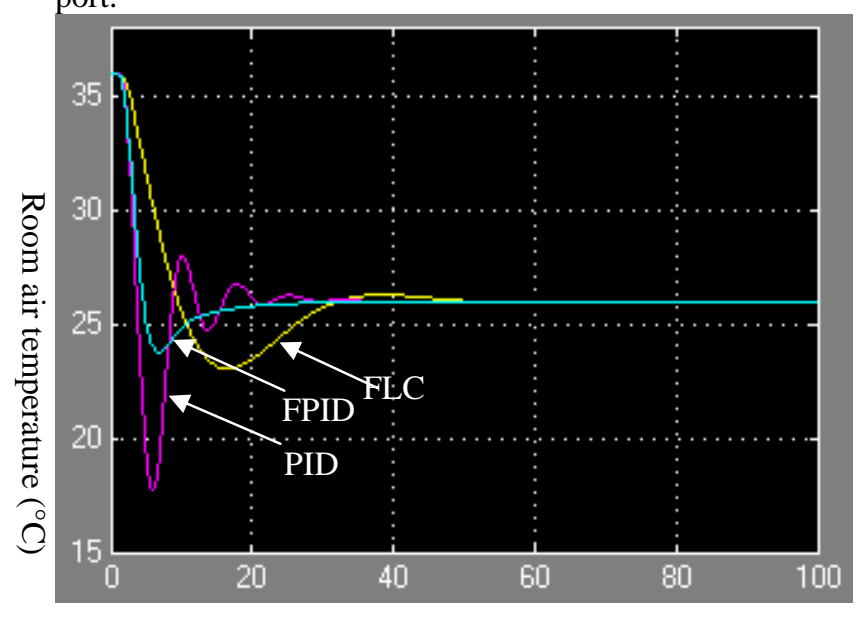

Time (s)

Fig. 5: Comparison of temperature response with disturbance.

\section{Conclusions}

In this paper, a novel two-stage FPID controller for room air temperature control in VRV system is proposed. The salient feature of the proposed controller is that it does not require an accurate model of the controlled plant, and the design process is lower than that of the other FPID controllers. Moreover, it has a simple structure and is easy to implement, which makes it ideally useful for the real VRV system. The two-stage FPID controller is tested in a triple evaporator VRV air conditioning system to demonstrate its set point tracking performance and disturbance rejection under different operating conditions. Simulation results show that the proposed controller is very effective and guarantees good robust performance against different disturbances and is better than those of the conventional FLC and PID controllers. Thus, it is recommended the promising control scheme to generate excellent and reliable temperature control quality in VRV system. 


\section{Acknowledgement}

This work is partially supported by National High Technology Research and Development Program of China (Grant No. 2006AA05Z238).

\section{References}

[1] F.C. Lin, Adaptive fuzzy logic-based velocity observer for servo motor drives. Mechatronics, 13:229-241, 2003.

[2] M.K. El-Sherbiny, G. El-Saady and A.M. Yousef, Efficient fuzzy logic load frequency controller. Energy Convers Manage, 43:1853-1863, 2002.

[3] Z.W. Woo, H.Y. Chung and J.J. Lin, A PID type fuzzy controller with self-tuning scaling factors. Fuzzy Sets, 115:321-326, 2000.

[4] D.D. Kukolj, S.B. Kuzmanovic and E. Levi, Design of a PID-like compound fuzzy logic controller. Eng Appl Artif, 14:785-803, 2001.

[5] J. Carvajal, G. Chen and H. Ogmen, Fuzzy PID controller: design, performance valuation, and stability analysis. Inform Sci, 123:249-270, 2000.

[6] M.J. Adams, Genetic multi-stage fuzzy PID controller with fuzzy switch. Proceeding of the IEEE international conference on systems, man and cybernetics, pp. 2239-2244, 2001.

[7] M.J. Adams, Multi-stage fuzzy PID controller with fuzzy switch. Proceeding of the IEEE international conference on control applications, pp. 323-327, 2001.

[8] M.J. Adams, Intelligent control of a direct-drive robot using multi-stage fuzzy logic. Midwest symposium on circuits and systems, pp. 543-546, 2001.

[9] C. Wu, Research on the characteristic and control strategy of multi-evaporator VRV air conditioning system. Master thesis. Shanghai JiaoTong University, China, 2002.

[10] C. Wu, Development of control method and dynamic model for multi-evaporator air conditioners (MEAC). Energy Conversion and Management, 46:451-465, 2005.

[11] J. Xia and E Winandy, Experimental analysis of the performances of variable refrigerant flow systems. Building Serv. Eng. Res. Technol, 25:17-23, 2004. 